\title{
A case of aplasia cutis congenita following fetal reduction of triplet pregnancy conceived through in vitro fertilization
}

\author{
Sae Yun $\mathrm{Kim}^{1 \oplus}$, Eun Sun $\mathrm{Kim}^{2 \oplus}$, Joonhong $\mathrm{Min}^{3 \oplus}$, Kyungyul Lee ${ }^{4 \oplus}$ \\ ${ }^{1}$ Department of Pediatrics, Yeouido St. Mary's Hospital, The Catholic University of Korea, Seoul, Korea; Departments of ${ }^{2}$ Pediatrics, \\ ${ }^{3}$ Dermatology and ${ }^{4}$ Pathology, Kangwon National University School of Medicine, Chuncheon, Gangwon-do, Korea.
}

\begin{abstract}
Background. Aplasia cutis congenita (ACC) is a rare congenital localized skin defect that is mostly diagnosed in the newborn or infant period. ACC type 5 often involves the trunk or extremities accompanied by fetus papyraceous (FP) or placental infarcts. The etiology and pathogenesis of this rare type of ACC are not well known. In this case, we report an ACC type 5 with a definite etiology.

Case. We report a preterm infant with ACC type 5, with diffuse bilateral leg lesions found at birth. He was the first baby of dichorionic twin after reduction from a dichorionic triplet pregnancy conceived through in vitro fertilization. A fetus papyraceous was found in juxtaposition with the affected baby's placenta. After 37 days of hospitalization, his leg lesions were successfully epithelized with supportive care. He is regularly visiting the Dermatology clinic for scar care and shows normal development without motor limitation.

Conclusions. Herein, we present a preterm infant with ACC type 5 and the placental pathology with fetus papyraceous of the artificially reduced monochorionic co-twin of the affected infant. We suggest a precautious decision in multifetal pregnancy reduction (MFPR) in dichorionic triplets, presenting ACC type 5 as an adverse outcome of MFPR.
\end{abstract}

Key words: aplasia cutis congenita, fetus papyraceous, multifetal pregnancy reduction, in vitro fertilization.

Aplasia cutis congenita (ACC), defined as the absence of all skin layers at birth, results from disrupted development or degeneration of skin in utero. In 1867, Frieden classified this congenital skin defect into nine groups based on the distribution of the affected area, associated anomalies, and the mode of inheritance. ${ }^{1}$ To date, no specific genetic focus is known related to ACC, however, ACC can be related to some genetic or malformation syndromes, such as Adams-Oliver syndrome, Bart syndrome, trisomy 13, trisomy 18 , monosomy 4, ectodermal dysplasia, and Johanson-Blizzard syndrome. ${ }^{1,2}$ Differential diagnosis of ACC includes focal dermal hypoplasia, dermoid cyst, encephalocele, epidermolysis bullosa, infections (herpes or varicella zoster virus), amniotic band

$凶 \quad$ Eun Sun Kim

naivesun1@hanmail.net

Received 11th June 2020, revised 13th February 2021, accepted 3rd March 2021. disruption syndrome or birth trauma ${ }^{3}$, thus, a thorough examination of the patient and history of familial skin defect, infection, or any birth trauma are important for ACC diagnosis.

Feus papyraceous (FP) formed by mummification of a dead fetus usually occurs during intrauterine death of a co-twin during the late first to early second trimester, whereas earlier or later death of a co-twin typically leads to complete resorption or maceration, respectively.,

We report a preterm neonate with bilateral symmetric ACC mainly on the lower extremities with FP who was born from a dichorionic triplet pregnancy conceived through in vitro fertilization (IVF) that had been artificially reduced to a dichorionic twin pregnancy. We focused on symmetric lower extremity ACC as a consequence of the development of advanced reproductive medicine. 


\section{Case Report}

A baby boy weighing $1,990 \mathrm{~g}$ was born at $35^{+2}$ weeks gestation with an unexpected large skin defect in both the legs and trunk. This had been an IVF pregnancy in which three embryos were transferred, two of which survived and one of which split, resulting in two monochorionic fetuses and a third dichorionic fetus. Chorionic villi sampling at 12 weeks gestation demonstrated that they were normal karyotypes. Radiofrequency ablation of the umbilical cord of one fetus was performed at 14 weeks, resulting in a dichorionic twin pregnancy. Afterward, the pregnancy was uneventful until preterm labor pain occurred. The mother was hospitalized due to preterm labor, and cesarean section was performed due to fetal deceleration. The baby boy's Apgar scores were 8 and 9 at 1 and 5 minutes, respectively, and he was cared for in the neonatal intensive care unit of the hospital where he was born.

The parents were examined by special tests including karyotyping before the pregnancy due to recurrent abortion (G2P0), but the result was normal. The mother's age was 39 years, and she did not take any special medication during this pregnancy. There was no family history of ACC, vascular abnormalities, vesiculobullous disease, or thrombophilia.
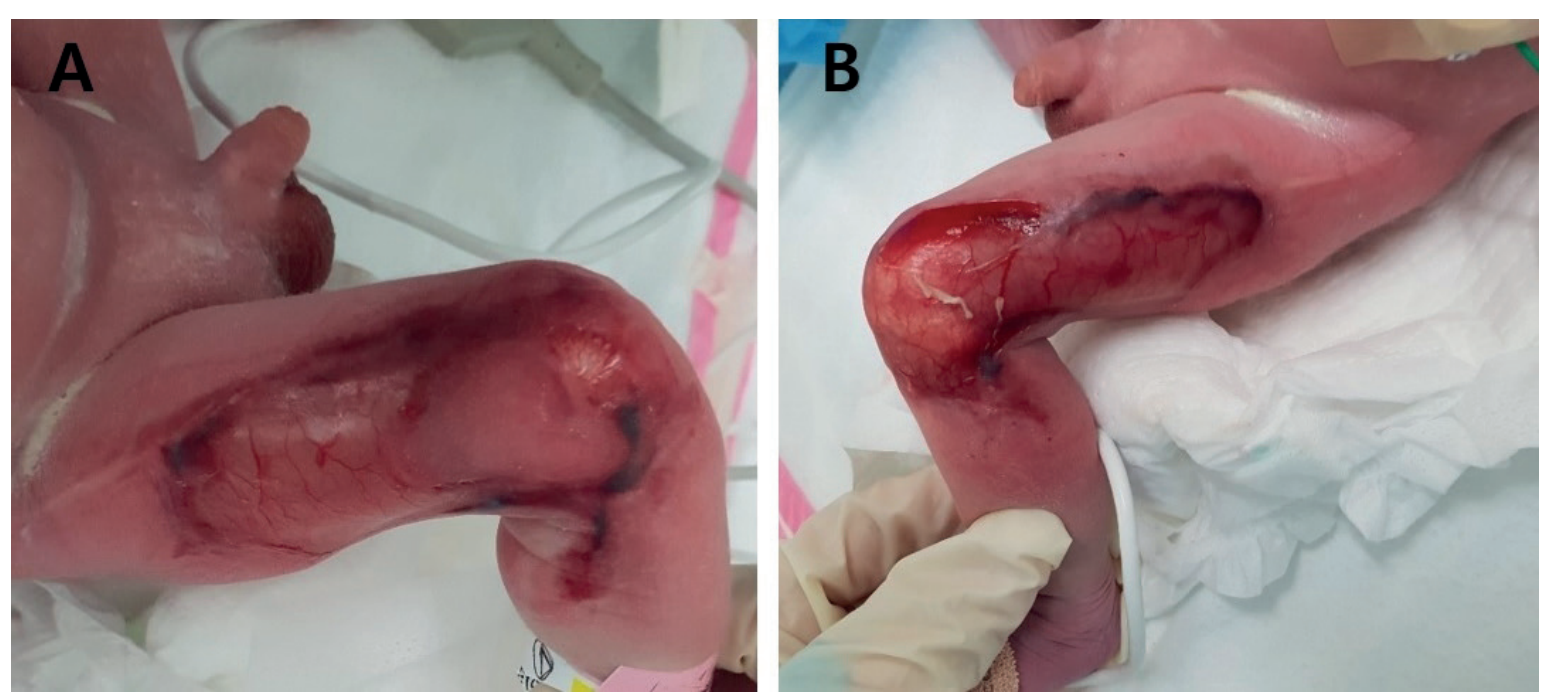

Fig. 1. Symmetric skin defects in both legs of the affected baby on the 1st day of life. (A) Lateral view of right

leg. (B) Lateral view of left leg.

Physical examination on the first day of life revealed well-demarcated ulcers in a symmetric distribution on both legs. Thin, silky, and transparent membranes were present, and small vessels were visible in both lesions (Fig. 1). Other abnormalities or any dysmorphic features were absent. Without specific diagnostic tests, ACC was diagnosed based on the clinical manifestation. The other live twin was a completely unaffected baby girl with a birth weight of 1,920 g. Formal pathological examination of the placenta after delivery revealed dichorionic and diamniotic twin placentas with microcalcification and a mummified fetus in one chorion (Fig. 2).

No skin biopsy was performed. Based on the clinical manifestation, a diagnosis of ACC type 5 was made. The patient's wounds were treated with a non-adhesive dressing with a pain controller (oral acetaminophen). After the lesions were washed with normal saline, mupirocin ointment (Esrovan Ointment ${ }^{\circledR}$ [JW Shinyak, Seoul, Korea]) and recombinant human epidermal growth factor ointment (Easyef Oint ${ }^{\circledR}$ [Daewoong Pharmaceutical Company, Seoul, Korea]) were applied. Afterward, the dressing area was covered with Physiotulle ${ }^{\circledR}$ [Coloplast A/S, Denmark], medifoam (Medifoam ${ }^{\circledR}$ [Ildong Pharmaceutical Company, Seoul, Korea]) was applied, and the pressure bandage was lightly 
fastened to prevent pressure from being applied to it. Because of the large skin defects, active fluid management and prophylactic antibiotic treatment were performed for the first 10 days until minimal epithelization was completed.
After one month of dressing in collaboration with the dermatologist and plastic surgeon, the baby's skin lesions were successfully epithelized (Fig. 3 a,b), and he was discharged after 37 days of hospitalization.
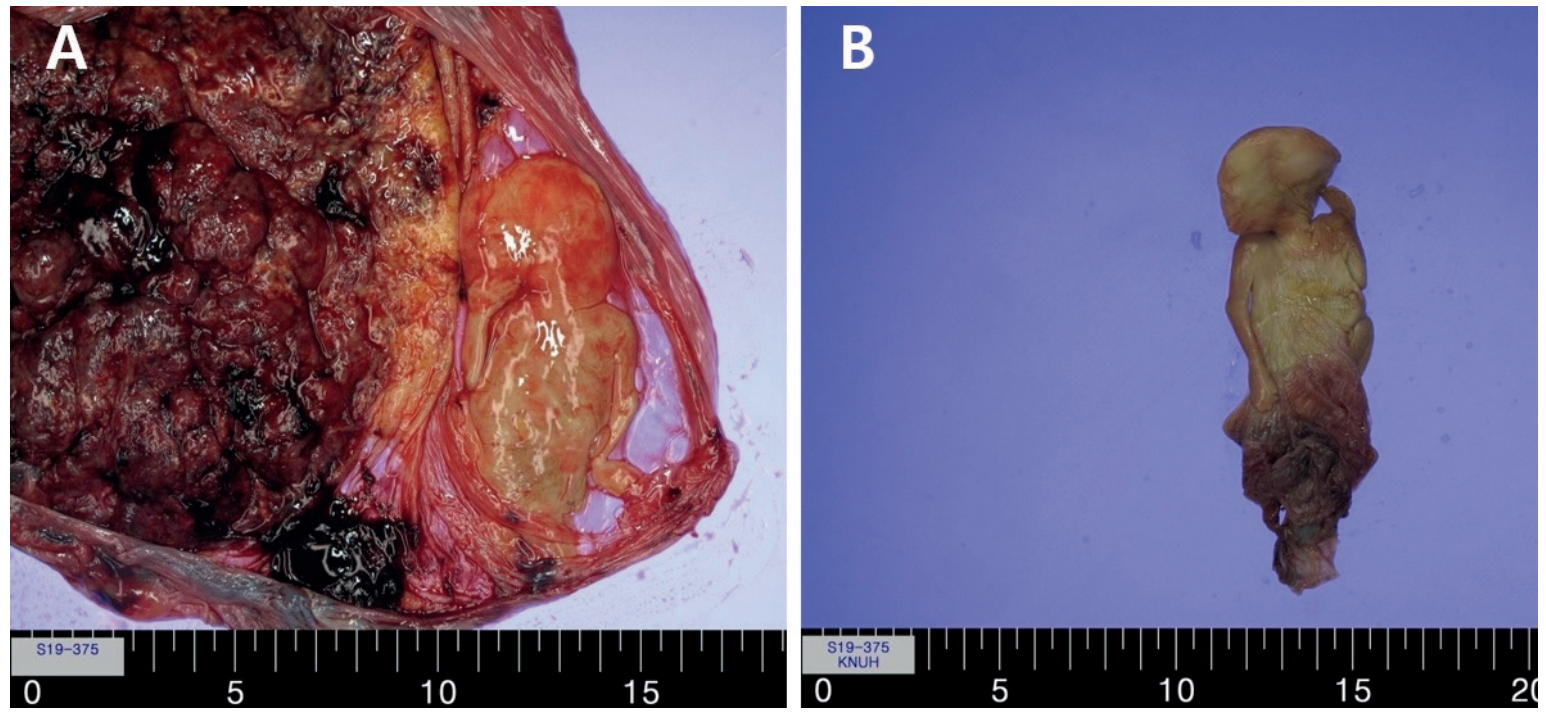

Fig. 2. Placental pathology of the pregnancy. (A) Fetus papyraceous in juxtaposition with the affected baby's placenta. (B) Detached fetus papyraceous with detailed structure.
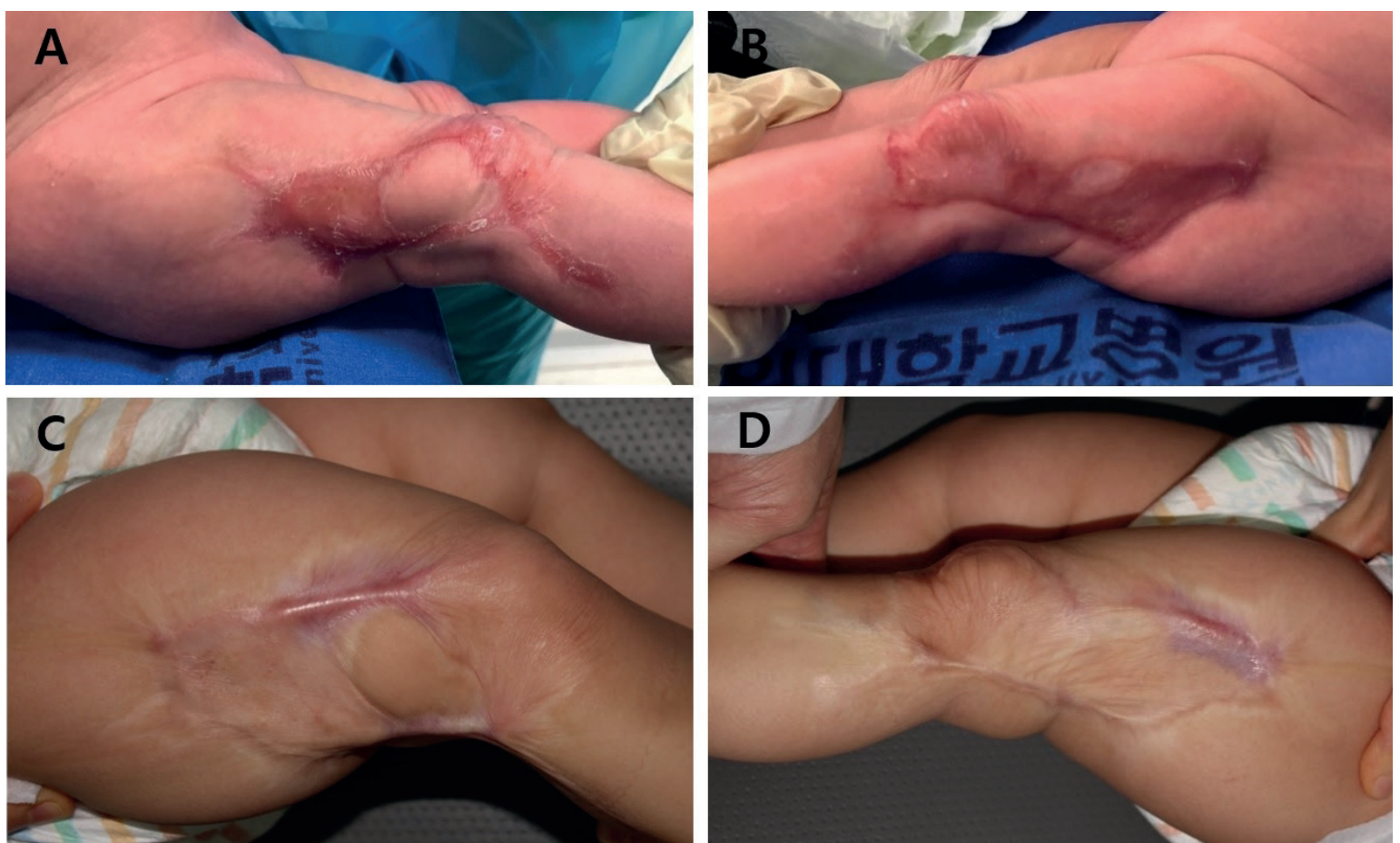

Fig. 3. Progression of aplasia cutis congenital lesions. (A, B) Epithelialized skin defects of both legs at 37 days of life (at hospital discharge). (C, D) Atrophic scar formation of both legs (at 12 months of age). 
The infant was followed up at dermatology and pediatrics outpatient clinics. At 12 months of age, the infant showed progressed atrophic scars in both legs (Fig. 3 c,d); thus, treatment with 5-fluorouracil and triamcinolone injection was started, further requiring scar intervention, such as skin grafting. Additionally, he showed normal developmental status per the Korean Developmental Screening Test and level 1 of the Gross Motor Function Classification System without any contracture or motor limitation.

Written informed consent was obtained from the patient's legal guardian for the publication of any potentially identifiable images or data included in this article.

\section{Discussion}

The incidence of ACC with FP is likely to be underreported because milder cases often go undetected and because the disappearance of an intrauterine twin at an early stage could be reported as a singleton pregnancy. A recent literature review in Korea revealed only 2 cases of ACC type 5 (ACC with FP or placental infarcts) per the Frieden classification ${ }^{1}$ among 59 reported ACC cases in Korea ${ }^{3}$, which explains the rarity of the ACC type 5 diagnosis.

The diagnosis of ACC is clinically determined in most cases and biopsies are not routinely performed. Based on the clinical manifestation of a skin defect after birth, ACC type 5 is diagnosed if the following three factors are met: 1) multiparous pregnancy, 2) placental vascular twin anastomoses, and 3) early twin death (FP). ${ }^{6}$ A review of 44 cases of ACC with FP found that the mean gestational age of fetal death was 13.3 weeks, with an approximately $1: 1$ ratio of female to male infants, and the maternal condition was normal prior to delivery in the majority of cases. ${ }^{7}$

Although the exact pathogenesis is unknown, several theories have been proposed regarding skin defects in ACC with FP. Among them, two hypotheses are most plausible. The first is feto- fetal transfusion; the decreased blood pressure of a dying twin can lead to a blood shift from the surviving twin through the shunt to the dying twin. The surviving twin may have hypovolemia and hypotension resulting in ischemia of endorgans such as the skin, sometimes have a watershed area. ${ }^{8}$ The second theory is that a dead fetus may pass thrombogenic materials to the viable twin through vascular anastomoses, and the coagulation cascade can be activated in the viable twin, causing ACC and any placental abnormalities. ${ }^{7}$ The premature calcifications in our case may reflect placental vascular insufficiency. It is also speculated that any brain injury may occur due to emboli through incomplete anastomosis vessel occlusion after laser ablation. Fortunately, the infant in our case showed normal findings on brain sonography and presented normal development at pediatric and rehabilitation outpatient clinics at 12 months of age.

The incidence of multiple gestation in South Korea has increased sharply over the past two decades (from $1.7 \%$ to $4.2 \%$ of total births between 2000 and 2018), reflecting a trend toward older maternal age and increased use of infertility treatment. Multifetal pregnancy reduction (MFPR) is a procedure that reduces higher-order pregnancies and was originally intended to improve perinatal outcomes. Typically, in dichorionic triamniotic (DCTA) triplet pregnancies, such as our case, the parents wish to retain a non-monochorionic twin pair after obstetric counselling. Miscarriage or preterm birth are known substantial risks of MFPR; however, an ACC with surviving cotwins is an unexpected event. One case series reported ACC incidence of $8 \%$ ( 2 of 26 newborns after laser ablation at 13 and 14 weeks) after MFPR. ${ }^{10}$ One similar case report suggested that the mechanism of ACC was laser burn ${ }^{11}$; however, a baby born after spontaneous cotwin loss during a MCDA pregnancy presented similar truncal and thigh lesions characteristic of ACC type $5,^{12}$ thereby disproving laser burn as the plausible cause. 
The assisted reproductive technology (ART) registry of 17 European countries revealed that the multiple delivery rate is approximately $20 \%$ after ART. ${ }^{13}$ In Korea, under the Korean guidelines, three-embryo transfers are the most frequent transfer number in IVF, and $29.8 \%$ of all IVF deliveries are twin or triplet deliveries. ${ }^{14}$ With the increase in multiple pregnancies, indications for MFPR may exist, especially in DCTA triplet pregnancies; however, whether MFPR in DCTA triplet pregnancy is superior to expectant management in terms of miscarriage or preterm birth is unknown. ${ }^{15}$ Furthermore, this report highlights a distinctive adverse outcome, namely, ACC type 5 with FP, after MFPR. Thus, we suggest that the decision for MFPR in DCTA triplet pregnancy be made with caution, and we present ACC type 5 as an adverse outcome of MFPR in DCTA triplet pregnancy.

\section{Acknowledgment}

The parents who consented with the study are gratefully appreciated.

\section{Author contribution}

The authors confirm contribution to the paper as follows: study conception and design: SYK, ESK; data collection: SYK, ESK, JM, KL; analysis and interpretation of results: SYK, ESK, JM, KL; manuscript preparation: SYK, ESK. All authors reviewed and approved the final version of the manuscript.

\section{Source of funding}

This study was supported by 2019 Research Grant from Kangwon National University.

\section{Conflict of interest}

The authors declare that there is no conflict of interest.

\section{REFERENCES}

1. Frieden IJ. Aplasia cutis congenita: a clinical review and proposal for classification. J Am Acad Dermatol 1986; 14: 646-660. https://doi.org/10.1016/S01909622(86)70082-0

2. Brackenrich J, Brown A. Aplasia Cutis Congenita. In: StatPearls [Internet]. Treasure Island (FL): StatPearls Publishing; 2021. Available from: https://www.ncbi. nlm.nih.gov/books/NBK535403/

3. Yang MY, Ha DL, Kim HS, Ko HC, Kim BS, Kim MB. Aplasia cutis congenita in Korea: single center experience and literature review. Pediatr Int 2020; 62: 804-809. https://doi.org/10.1111/ped.14192

4. Mannino FL, Jones KL, Benirschke K. Congenital skin defects and fetus papyraceus. J Pediatr 1977; 91: 559564. https://doi.org/10.1016/S0022-3476(77)80502-7

5. Landy HJ, Keith LG. The vanishing twin: a review. Hum Reprod Update 1998; 4: 177-183. https://doi. org/10.1093/humupd/4.2.177

6. Sobczak A, Tomasik T, Zając A, Klasa B, Kruczek P, Kwinta P. New features of aplasia cutis congenita type 5 - Skin atrophy associated with respiratory insufficiency and multiple intestinal atresia caused by the early death of twin fetus. Pediatr Neonatol 2019; 60: 473-474. https://doi.org/10.1016/j. pedneo.2018.09.004

7. Tempark T, Shwayder TA. Aplasia cutis congenita with fetus papyraceus: report and review of the literature. Int J Dermatol 2012; 51: 1419-1426. https:// doi.org/10.1111/j.1365-4632.2012.05545.x

8. Schaffer JV, Popiolek DA, Orlow SJ. Symmetric truncal aplasia cutis congenita following multifetal reduction of a sextuplet pregnancy. J Pediatr 2008; 153: 860-863. https://doi.org/10.1016/j. jpeds.2008.07.009

9. Korean Statistical Information Service. Birth statistics. Available from: http://www.kosis.kr (Accessed on 2 March 2020).

10. O’Donoghue K, Barigye O, Pasquini L, Chappell L, Wimalasundera RC, Fisk NM. Interstitial laser therapy for fetal reduction in monochorionic multiple pregnancy: loss rate and association with aplasia cutis congenita. Prenat Diagn 2008; 28: 535543. https://doi.org/10.1002/pd.2025

11. Mugarab-Samedi V, Lodha A, ElSharkawy A, Al Awad E. Aplasia cutis congenita as a result of interstitial laser therapy for fetal reduction in monochorionic twins: Conservative approach and outcome. Int J Surg Case Rep 2017; 41: 68-70. https:// doi.org/10.1016/j.ijscr.2017.10.007 
12. Snyder ML, Ilyas H. Type V aplasia cutis congenita with fetus papyraceus. JAAD Case Rep 2019; 5: 303305. https://doi.org/10.1016/j.jdcr.2019.02.015

13. European IVF-Monitoring Consortium (EIM); European Society of Human Reproduction and Embryology (ESHRE), Kupka MS, et al. Assisted reproductive technology in Europe, 2011: results generated from European registers by ESHRE. Hum Reprod 2016; 31: 233-248. https://doi.org/10.1093/ humrep/dev319
14. Committee for Assisted Reproductive Technology, Korean Society of Obstetrics and Gynecology, Choi YM, Chun SS, et al. Current status of assisted reproductive technology in Korea, 2009. Obstet Gynecol Sci 2013; 56: 353-361. https://doi.org/10.5468/ ogs.2013.56.6.353

15. Anthoulakis C, Dagklis T, Mamopoulos A, Athanasiadis A. Risks of miscarriage or preterm delivery in trichorionic and dichorionic triplet pregnancies with embryo reduction versus expectant management: a systematic review and meta-analysis. Hum Reprod 2017; 32: 1351-1359. https://doi.org/10.1093/humrep/dex084 